\title{
Review
}

\section{Renal disease in patients with Crohn's disease}

\author{
Linda Atencio Ibarra 1,2, Roberto Navarro Quiroz ${ }^{3}$, Gustavo Aroca Martinez 1,2, Joany Sarmiento \\ Gutierrez 1,2, Andres Cadena Bonfanti 1,2, Leandro Luis Sierra Carrero ${ }^{4}$, Lorena Gomez Escorcia 1,2, \\ Roberto Garcia Alzate 6,7, Maria Ospino Rodriguez 1,2, Daisy Lozano Arias 7, Henry Gonzalez \\ Torres 1, Carmen Carrero Gonzalez ${ }^{1}$, Cecilia Fernandez Ponce8, and Elkin Navarro Quiroz 1,7,* \\ 1 Faculty of Basic and Biomedical Sciences, Universidad Simon Bolivar, Barranquilla, 080001, Colombia; \\ 2 Department of Nephrology, Clinica de la Costa, Barranquilla, 080001, Colombia; \\ 3. CMCC-Centro de Matemática, Computação e Cognição, Laboratório do Biología Computacional e \\ Bioinformática-LBCB, Universidade Federal do ABC, Sao Paulo, 01023, Brazil; \\ 4. Department of Science, Universidad del Norte, Barranquilla, 080001, Colombia; \\ 5 Faculty of Basic Sciences, Universidad Libre, Barranquilla, 080001, Colombia; \\ 6 Faculty of Basic Sciences, Universidad del Atlántico, Barranquilla, 080001, Colombia; \\ 7 School of Medicine, Universidad San Martin, Barranquilla, 080001, Colombia; \\ 8. Universidad de Cadiz, Department of Biomedicine, Biotechnology and Public Health, Cadiz, Spain \\ * Correspondence: enavarro26@unisimonbolivar.edu.co; Tel.: +57-3015987517
}

\begin{abstract}
Crohn's disease (CD) results from an aberrant immune response against the commensal microbiota in genetically susceptible hosts. However, the nature of the immune defects, the microflora involved and the genetic susceptibility remain incompletely defined and controversial. Extraintestinal manifestations occur in up to $25-35 \%$ of patients and generally precede the onset of gastrointestinal symptoms, which are often of a colonic nature and are influenced by disease activity. Renal manifestations can be considered dependent on the same immune mechanism that determines inflammatory bowel disease in $\mathrm{CD}$. This review seeks to describe the current state of association between $\mathrm{CD}$ and kidney disease.
\end{abstract}

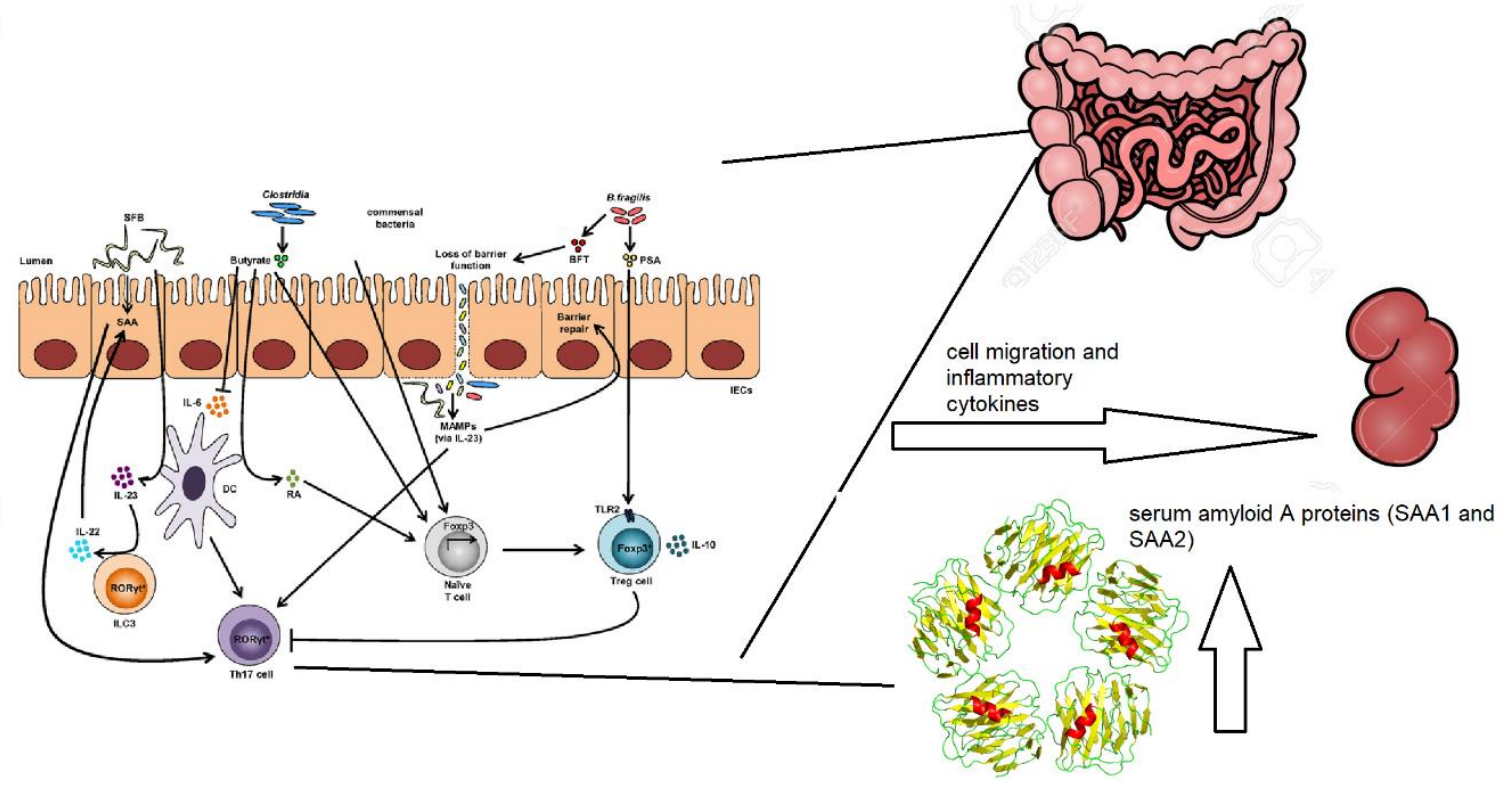

Keywords: Crohn's disease; renal disease; amyloidosis

\section{Introduction}


Crohn's disease $(C D)$ is a degenerating systemic inflammatory disease that principally affects the gastrointestinal tract with extraintestinal effect and is associated immune illnesses. Genome-wide association studies have identified susceptibility loci that have been related to $C D$, also have identified environmental factors, lead to innate immune responses and adaptive responses to a diminished diversity of commensal microbiota [1].

Among the pathological manifestations of $\mathrm{CD}$, the formation of kidney stones is usually more common in affected individuals than in the general population. The frequency of lithiasis in this group of patients is estimated to be $12 \%-28 \%$ [2]. Often these stones are calcium oxalate (CaOx), which previously has been recognized in elevated urinary oxalate output that associated fat and bile salt malabsorption. Influences other than urinary supersaturation with $\mathrm{CaOx}$ have also been implicated in stone development, e.g., low levels of the antilithogenic agents, magnesium, and citrate[3], [4]. Although, patients with CD have a tendency to develop hyperoxaluria, hypocitraturia, hypomagnesuria and hypophosphaturia and have a low volume of urine[5], Paradoxically, this patients with $\mathrm{CD}$ do not develop kidney stones, so the factors that determine the risk that a $\mathrm{CD}$ with some type of urological disorder is still a cause for discussion [6],[7].

Additionally, the appearance of amyloidosis has been designated in patients with $\mathrm{CD}$; It is considered for the reactivity in the acute phase that is performed during sustained inflammation, such as that observed in several other chronic inflammatory diseases (mainly rheumatic but inflammatory bowel disease [IBD]). Amyloidosis is a rare but serious complication of IBD, being more frequent in patients with CD than in those with ulcerative colitis (UC) [8].

This review seeks to describe the present state of association between $\mathrm{CD}$ and renal disease; moreover, we highlight the convergence of $\mathrm{CD}$ with amyloidosis that can trigger the sustained inflammation that may explain the pathological alteration observed in both diseases.

\section{Materials and Methods}

Searches were performed in PubMed, PubMed Central (PMC), and Web of Science using the following MESH search terms: "Crohn's disease" and "renal disease." For PubMed and PMC, the R RISmed package was used [9]. Systematic searches were performed according to PRISMA criteria, obtaining 1002 records (from 2009 to 2019). The advanced search tool was used for Web of Science (using the Boolean operator AND among the previously mentioned terms, obtaining 415 records, from 2009 to 2019). Then the relevance of all records was evaluated according to the title and summary. If considered relevant, the entire article was examined, and 51 articles were selected using this method.

\subsection{Histopathological spectrum of renal biopsies in patients with Crohn's syndrome}

Renal disease is a complication of IBD, including $\mathrm{CD}$ and $\mathrm{UC}$; renal and genitourinary involvement has been reported in $4 \%-23 \%$ of patients with CD [10]manifesting primarily as urinary stones, fistulas, and renal tubular damage. Parenchymal renal disease is rare but has been well documented in global literature as case reports describing glomerulonephritis (GN)[6].

In patients with $\mathrm{CD}$, the most common indication for renal biopsy was acute or chronic kidney failure (63\% [52 of 83]) and nephrotic-range proteinuria (16\% [13 of 83]). IgA nephropathy (IgAN) was the most common diagnosis (24\% [20 of 83]), followed by interstitial nephritis (19\% [16 of 83]), arterionephrosclerosis (12\% [10 of 83$]$ ), acute tubular injury ( $8 \%$ [7 of 83$]$ ), proliferative GN (7\% [6 of $83]$ ), and minimal change disease (5\% [4 of 83]) [11].

The frequency of IgAN in specimens of patients with IBD was significantly higher than that in all other native renal biopsy specimens from the same period ( $24 \%$ [20 of 83 ] versus $8 \%$ [2734 of 33,630]; $\mathrm{P}<0.001$ ) [11]. 

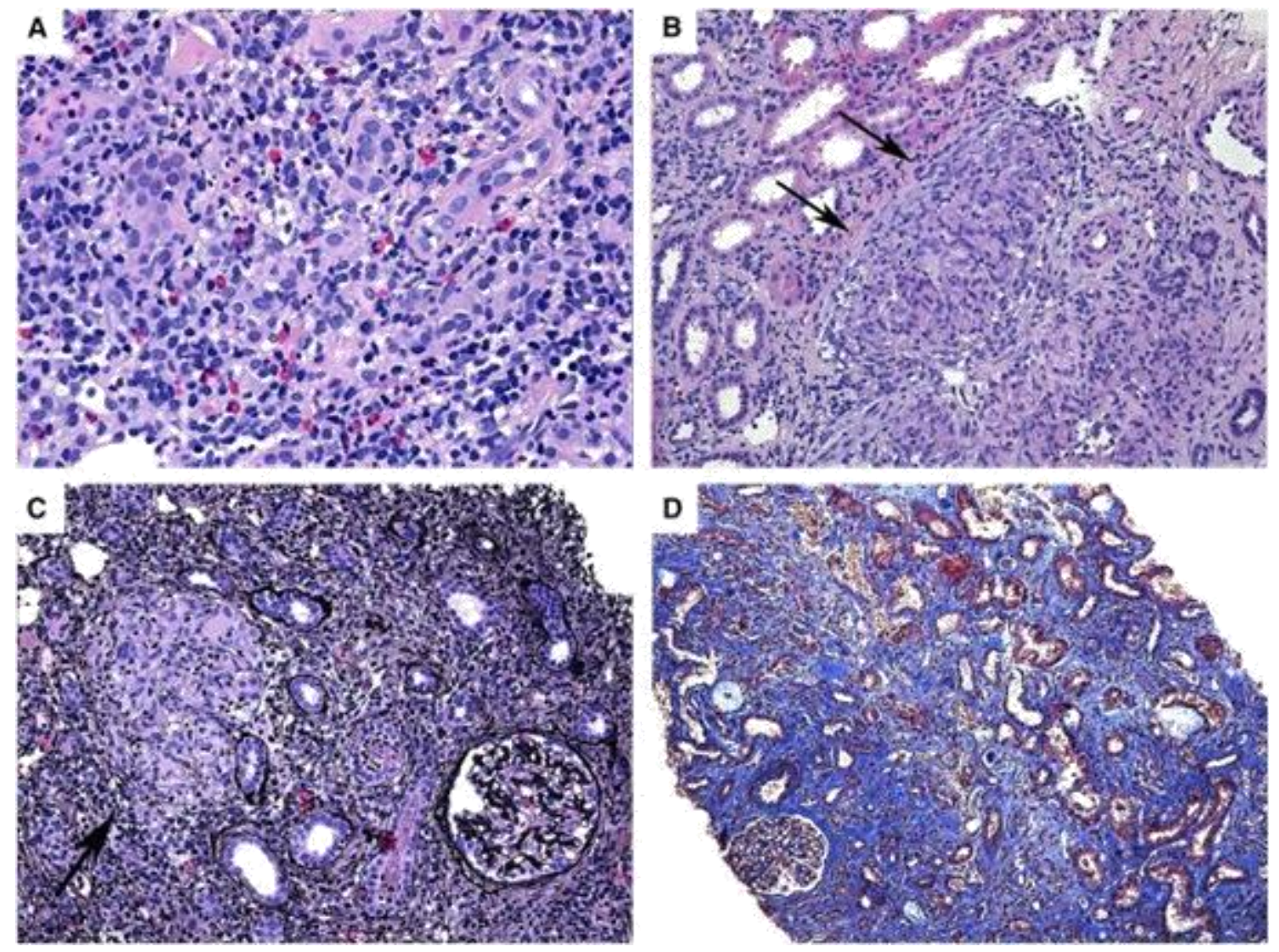

Figure 1. Pathological features of interstitial nephritis associated with inflammatory bowel disease: (a) Acute interstitial nephritis with dense interstitial inflammation predominantly comprising lymphocytes and eosinophils (hematoxylin and eosin, original magnification $\times 200$ ); (b and $\mathbf{c}$ ) Granulomatous interstitial nephritis with interstitial infiltration by mononuclear cells and noncaseating granulomas with multinucleated giant cells (arrows) (hematoxylin and eosin, Jones methenamine silver, both original magnification $\times 200$ ); (d) Chronicinterstitial nephritis with diffuse interstitial fibrosis and tubular atrophy; the glomerulus is preserved (Masson trichrome, original magnification $\times 100$ ) modified from [11].

In summary, $\mathrm{CD}$ is associated with a spectrum of renal complications likely related to chronic inflammation or drug therapy in these patients. Additionally, the most common diagnosis on renal biopsy is IgA nephropathy, and its significantly higher prevalence in patients with CD suggests a shared pathophysiology between the intestinal and renal disease.

Interstitial nephritis is the second most common diagnosis, which is often, although not always, associated with amino salicylate therapy. In general, a high degree of clinical suspicion is needed for the early diagnosis and prevention of these renal complications of CD[11].

\subsection{MicroRNAs in patients with Crohn's disease who develop renal disease}

Emerging evidence suggests that microRNA (miRNA)-mediated gene regulation influences various autoimmune disease processes, including CD, but the biological function of miRNAs in CD remains debatable. miRNAs are a group of endogenous, small, noncoding RNAs approximately 22 nucleotides in length that regulate gene expression by binding to the noncoding region (3-UTR) of target mRNA molecules at the posttranscriptional level. Interestingly [12], there is compelling evidence that a range of miRNAs are involved in the regulation of immunity, including the development and differentiation of immune cells, and the release of inflammatory mediators. miRNAs are differentially expressed in the serum and mucosa of patients with CD, suggesting that miRNA deregulation can play an important role in the development of CD [12]. 
miR-141 is aberrantly expressed in two other autoimmune diseases (lupus and psoriasis) [10]. It has been reported that several factors, such as Wnt signaling, DNA methylation, and miRNAs, contribute to the regulation of The chemokine (C-X-C motif) ligand (CXCL); however, the mechanism that promotes CXCL expression during intestinal inflammation remains unclear [11].

Based on the fact that CXCL12 $\beta$ levels significantly increase in CD and that miR-141 shows a significant reduction of expression in the same cell type, we assume that miR-141 targeting the CXCL12 $\beta$ pathway in CD serves as an important mechanism to regulate the colonic expression of

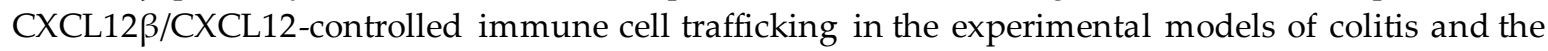
process of CD dysfunction in humans[13].

Additionally, it was previously observed that the traffic of immune cells mediated by miR-141 through CXCL12 $\beta$ in CD plays a key role in crohn's disease which may give some idea of how this mechanism can be regulated in these patients. [13].

\subsection{Interleukin-17 and Thelper 17 cells in patients with CD with renal disease}

Interleukin-17 (IL-17) is key in the regulation of immune response, and T helper 17 (Th17) cell responses are associated with chronic inflammatory diseases, so much so that the Th17 subset generally produces interleukin-17A, which In experimental models of organ-specific autoimmune inflammation, it plays an important role in CD [14].

Both CD and IBD local inflammation in the mucosa leads to the destruction of the lamina, resulting in complications such as perforations and internal or external fistulas[15] and when inflammation occurs in different locations, it is known as ulcerative colitis [16].

Inflammatory manifestations in joints other than those observed in rheumatoid arthritis (RA) can be a part of spondyloarthropathies. As in RA and psoriasis, the contribution of tumor necrosis factor (TNF) has been demonstrated in a significant proportion of patients with IBD by the positive effect of TNF inhibition [16].

A study in endoscopic biopsies has reported that cytokines IL-23 and IL-17, as well as IL-12, are overexpressed in CD lesions, have even shown that interferon $-\gamma$ is overexpressed on $C D$ and that expiration It may be associated with pathology in these patients, additionally, the secretion of IL-17A, as well as of IL-17F, IL-21, and IL-22, suggests that Th17 subset plays a crucial role as a pleiotropic proinflammatory Th subset [17].

There is experimental evidence to support the notion that Th1 and Th17 cells contribute to renal injury in renal inflammatory diseases like glomerulonephritis [18]. This suggests that the mechanisms of migration in Th1 and Th17 cells can be a problem for these patients, causing the inflammatory process to affect the kidneys.

\subsection{Immune cell migration in Crohn's disease in patients with renal disease}

The validity of the paradigm of immune cell migration in chronic diseases and the different organs affected by their deregulation is the key to understanding the association between CD and renal involvement [19]. On the other hand, patients with leukocyte adhesion deficiency synd rome have been observed to exhibit impaired leukocyte migration into tissues; this results in recurrent bacterial and fungal infections and peripheral leukocytosis due to defects at different stages in the multistep paradigm of leukocyte transit, including reduced expression of $\beta 2$ integrins or fucosylated selectin ligands or an impaired ability to activate leukocyte integrins after chemokine stimulation [20] Therefore, "experiments of nature" have provided a "proof of principle" that the transit of molecules involved in the different steps of the multistep transit paradigm in humans contributes in unique ways to leukocyte transit in vivo [21].

Natalizumab, a monoclonal antibody targeting the $\alpha 4$ integrin chain that blocks the binding of $\alpha 4 \beta 1$ (VLA-4) to VCAM-1 in Th1 cells (Th1 cell infiltration in the brain is mediated by the binding of $\alpha 4 \beta 7$ to CAM-1, with a very similar process occurring in the Th1cell infiltration in the intestines), has been successfully used in the treatment of multiple sclerosis and CD [20]. This clinical success has established the "proof of principle" that specifically interfering with the transit of leukocytes to the tissue is a new effective therapeutic strategy. 
However, complications in infectious diseases that led to the withdrawal of this promising therapy necessitate a greater understanding of the host defense components mediated by subsets of specific leukocytes and specific transit molecules, as well as the need to find a method more precise and accurate of specific leukocyte inhibitors in transit in cellular subsets, allowing the host response to an infection or acute injury to comprise a robust neutrophilic inflammatory response [22].

As is often the case in inflammation, this is a "double-edged sword," and this robust protective response can also be detrimental to the host tissue as host response to many acute tissue attacks, such as ischemia followed by reperfusion, induces a neutrophil-rich inflammatory response that substantially contributes to tissue injury [23]. Ischemia-reperfusion injury is believed to contribute to many important pathological conditions, including acute myocardial infarction, stroke, shock, and acute respiratory distress syndrome [24][25].

Th1 inflammation is characterized by tissue infiltration of interferon $-\gamma$ CD4 + and CD8 + secretory effector $\mathrm{T}$ cells and activated macrophages. This type of cell-mediated inflammatory response is thought to play an important pathophysiological role in a multitude of prominent human diseases, including type 1 diabetes mellitus, CD [26], multiple sclerosis, RA, atherosclerosis, psoriasis, and solid organ allograft rejection. Interfering with the trafficking of molecules in Th1 cells and activated macrophages will have the advantage of being potentially beneficial in many of the aforementioned disorders [27]. In fact, the inhibition of integrin $\alpha \mathrm{L}$ (CD11a), which together with CD18 forms the LFA-1 complex, has proven to be clinically promising with the use of odulimomab [28] for graft-versus-host-disease and transplant rejection and efalizumab for psoriasis [29]. In addition, natalizumab is very effective in CD and multiple sclerosis. However, blocking all $\alpha 4$ mediated leukocyte trafficking will lead to increased susceptibility to infection [30].

The challenge, then, is identifying the specific adhesion and signaling molecules that control the transport of these immune cells. Initially, it was thought that the CCR3 chemokine receptor was a molecule of this kind; however, subsequent studies have shown that, although expressed in all eosinophils, it is present only in a minority of Th2 cells[31]. Mice with CCR3 deficiency have a profound defect in eosinophil migration, but Th2 cell trafficking appears to be intact, and mice with CCR3 deficiency can generate a tissue-specific allergic response [32].

Therefore, CCR3 blocking is a feasible approach to blocking eosinophil transit, but not Th2 cell transit; Th2 cell transport is under the control of chemotactic receptors and genes inducible by the STAT6 transcription factor [33]. Therefore, Th1 cells use "preferably" CXCR3, CXCR6, and CCR5, whereas Th2 cells use "preferably" CCR4, CCR8, and the prostaglandin D2 receptor CRTH2. However, it remains to be determined whether the inhibition of these or other combinations of chemokine receptors specifically expressed in Th1 versus Th2 cells can selectively inhibit the transport of any type of $\mathrm{T}$ cell, either alone or in combination with integrin blocking therapy in patients with CD [34].

\subsection{Main pathophysiological effects of Crohn's disease that may be triggered by a renal pathology}

It generally is accepted that $\mathrm{CD}$ results from an aberrant immune response to commensal microflora in genetically susceptible individuals. However, the nature of immune defects, the microflora involved, and genetic susceptibility remain incompletely defined and controversial [35].

On the other hand, the most frequent renal complication in patients with $\mathrm{CD}$ is nephrolithiasis with an incidence of $4 \%-23 \%$ [36], followed by nephrotic syndrome [37] and 24-h urinary proteinuria > $10 \mathrm{~g}$ [38]. Kidney stone formation occurs in $12 \%$ of patients with CD [39].

Regarding the pathophysiological causes of $\mathrm{CD}$ with nephrotic syndrome, some researchers attribute it to an abnormal humoral immune response of Th2 or B cells, whereas some believe that CD is caused by a Th1 or Th17 immune reaction or an autoimmune abnormality [40], [41].

Additionally, the increased urinary excretion of orosomucoid (ORM, also called $\alpha-1$ glycoprotein acid) has been described, which can provide a new biomarker in CD. One study has reported that U-ORM/u-CREAT values were seven times higher in children $(0.50 \mathrm{vs} 0.07 \mathrm{mg} / \mathrm{mmol}$; $\mathrm{P}<0.001)$ and two times higher in adults $(0.32 \mathrm{vs} 0.14 \mathrm{mg} / \mathrm{mmol} ; \mathrm{P}=0.01)$ with active $\mathrm{CD}$ than in patients with inactive disease. U-ORM/u-CREAT has shown good correlation with conventional 
inflammatory markers (hs-CRP, serum ORM; $\mathrm{P}<0.01$ ) and activity indices (HBI, $\mathrm{P}=0.018$; PCDAI, $\mathrm{P}<0.001)$. Furthermore, U-ORM/u-CREAT has a discriminative performance similar to those of hsCRP and serum ORM in the differentiation of active and inactive CD in pediatric patients [42].

\subsection{Pathological characteristics of amyloidosis in Crohn's disease}

Amyloidosis is one of the complications that may occur in patients with CD. Some unusual characteristics of both amyloidosis and CD in these patients include the relatively advanced age of patients with CD but relatively young age of those with amyloidosis and the high incidence of colon involvement in the former. In addition, recurrent polychondritis, seronegative RA, and cutaneous vasculitis, which are not fortuitously associated with amyloidosis, can be observed in patients with $\mathrm{CD}$, with mainly CD with colon involvement observed in cases of seronegative RA and cutaneous vasculitis. The frequent associations of CD with pyoderma gangrenosum are also worth noting [43].

All of these systemic, skin, or gastrointestinal tract disorders associated with amyloidosis are characterized by tissue damage with inflammatory infiltrates, without clear evidence of an autoimmune mechanism, which could be part of an abnormal immune response against connective tissue components or infectious agents. It is also worth noting that a no fortuitous association has been suggested between $\mathrm{CD}$ and amyloidosis [43].

Acceleration of Amyloidosis by Inflammation is unclear but there is information that $\mathrm{T}$ helper 17 (Th17) cells, defined by ROR $\gamma \mathrm{t}$-dependent production of IL-17A and IL-17F, exert homeostatic functions in the gut upon microbiota-directed differentiation from naive CD4+ $\mathrm{T}$ cells. In the microenvironment the production of cytokines is controlled by serum amyloid A proteins (SAA1 and SAA2) secreted by adjacent intestinal epithelial cells. However, the performances of Th17 cells vary substantially agreeing to their environment. [44].

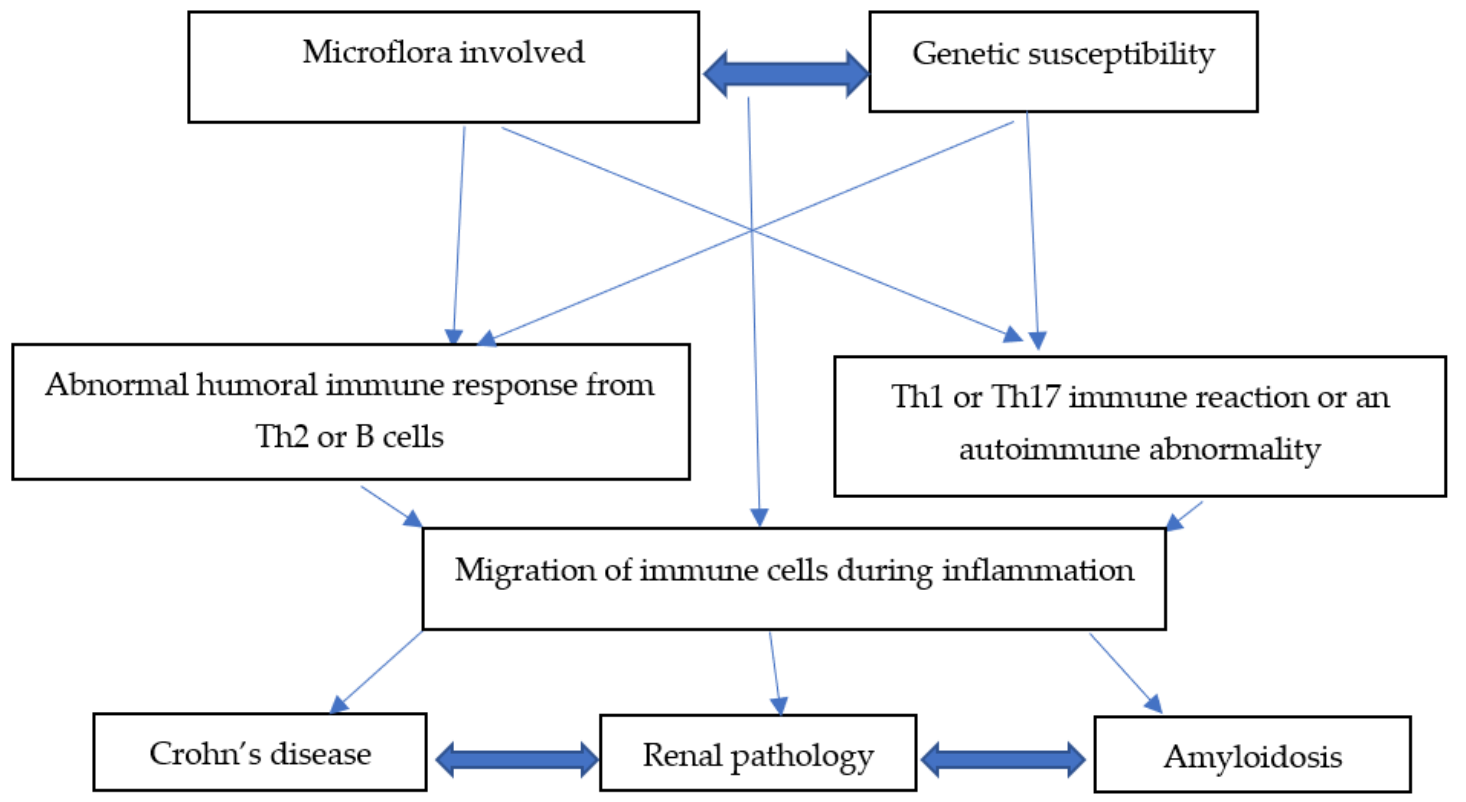

Figure 2. Pathological associations between $\mathrm{CD}$ and other complications such as amyloidosis and renal disease.

\section{Conclusions}

The association between renal pathological abnormalities and CD found within the spectrum of nephrotic syndrome to nephrolithiasis is variable; moreover, the underlying mechanisms that trigger renal pathologies in patients with $\mathrm{CD}$ remain debatable. However, they can be grouped into abnormal humoral immune response of Th2 or B cells and alterations in the migration mechanism of immune cells during inflammation in patients with CD. 
Likewise, alterations (amyloidosis) in the immune system in patients with $\mathrm{CD}$, results of the loss of the initial homeostasis probably caused by alteration in intestinal microbiota can collaborate to induce inflammation in other organs with the kidneys.

Author Contributions: Conceptualization, E.N.Q and R.N.Q.; methodology, E.N.Q., L.A.I., G.A.M, E.D.A.,H.G.T, D.L.A., R.G.A, M.O.R., C. and L.G.E.; writing - original draft preparation, E.N.Q., R.N.Q., E.D.A., A.C.B., J.S.G., and C.C.G., All authors contributed to the study design and preparation of the manuscript and have read and approved the final version.

Funding: We declare that the funds or sources of support received in this specific internal report study were from Simón Bolívar University, Barranquilla, Colombia and that the external funding was from the Ministery of Science, Technology, and Innovation of Colombia, subsidy 125380763038 and 125380763188 . We clarified that the funder had no role in the design of the study, in the collection and analysis of data, in the decision to publish, or in the preparation of the manuscript

Acknowledgments: Simón Bolívar University and Ministery of Science, Technology, and Innovation of Colombia for facilitating the resources for article publication

Conflicts of Interest: the authors state that they have no conflicts of interest

\section{References}

[1] D. C. Baumgart and W. J. Sandborn, “Crohn's disease," Lancet, vol. 380, no. 9853, pp. 1590-1605, Nov. 2012.

[2] N. McConnell, S. Campbell, I. Gillanders, H. Rolton, and B. Danesh, "Risk factors for developing renal stones in inflammatory bowel disease," BJU Int., vol. 89, no. 9, pp. 835-841, May 2002.

[3] M. P. Banner, "Genitourinary complications of inflammatory bowel disease.," Radiol. Clin. North Am., vol. 25, no. 1, pp. 199-209, Jan. 1987.

[4] J. D. Oliver, Z. S. Hammoudeh, A. A. Akhavan, and N. V. Tran, "Flap Reconstruction of Gastrointestinalto-Genitourinary Fistulas: A 20-Year Experience," J. Reconstr. Microsurg., vol. 35, no. 07, pp. 479-484, Sep. 2019.

[5] L. H. Smith, H. Fromm, and A. F. Hofmann, "Acquired Hyperoxaluria, Nephrolithiasis, and Intestinal Disease," N. Engl. J. Med., vol. 286, no. 26, pp. 1371-1375, Jun. 1972.

[6] E. Hylander, S. Jarnum, and I. Frandsen, "Urolithiasis and hyperoxaluria in chronic inflammatory bowel disease.," Scand. J. Gastroenterol., vol.14, no. 4, pp. 475-9, 1979.

[7] T. Alelign and B. Petros, "Kidney Stone Disease: An Update on Current Concepts," Advances in Urology, vol. 2018. Hindawi Limited, 2018.

[8] A. F. Brunger, H. L. A. Nienhuis, J. Bijzet, and B. P. C. Hazenberg, “Causes of AA amyloidosis: a systematic review," Amyloid, vol. 27, no. 1, pp. 1-12, Jan. 2020.

[9] S. Kovalchik, "RISmed: download content from NCBI databases," R Packag. version, vol. 2, no. 5, 2015.

[10] A. N. Manganiotis, M. P. Banner, and S. B. Malkowicz, "Urologic complications of Crohn's disease.," Surg. Clin. North Am., vol. 81, no. 1, pp. 197-215, x, Feb. 2001.

[11] J. M. Ambruzs, P. D. Walker, and C. P. Larsen, "The histopathologic spectrum of kidney biopsies in patients with inflammatory bowel disease.," Clin. J. Am. Soc. Nephrol., vol.9, no. 2, pp. 265-70, Feb. 2014.

[12] E. V. Makeyev and T. Maniatis, "Multilevel Regulation of Gene Expression by MicroRNAs," Science (80-. )., vol. 319, no. 5871, pp. 1789-1790, Mar. 2008.

[13] Z. Huang et al., "miR-141 Regulates colonic leukocytic trafficking by targeting CXCL12 $\beta$ during murine colitis and human Crohn's disease," vol. 63, pp. 1247-1257, 2014.

[14] P. Miossec, "IL-17 and Th17 cells in human inflammatory diseases," Microbes Infect., vol. 11, no. 5, pp. 625630, Apr. 2009.

[15] R. Panaccione, "Mechanisms of inflammatory bowel disease.," Gastroenterol. Hepatol. (N. Y)., vol. 9, no. 8, pp. 529-32, Aug. 2013.

[16] S. Menegatti, E. Bianchi, and L. Rogge, "Anti-TNF Therapy in Spondyloarthritis and Related Diseases, Impact on the Immune System and Prediction of Treatment Responses.," Front. Immunol., vol. 10, p. 382, 2019.

[17] Y. Yagi, A. Andoh, O. Inatomi, T. Tsujikawa, and Y. Fujiyama, "Inflammatory responses induced by interleukin-17 family members in human colonic subepithelial myofibroblasts," J. Gastroenterol., vol. 42, no. 9, pp. 746-753, Sep. 2007. 
[18] F. Azadegan-Dehkordi, N. Bagheri, H. Shirzad, and M. Rafieian-Kopaei, "The role of Th1 and Th17 cells in glomerulonephritis.," J. Nephropathol., vol. 4, no. 2, pp. 32-7, Apr. 2015.

[19] A. W. Segal, “Making sense of the cause of Crohn's - a new look at an old disease.," F1000Research, vol.5, p. 2510, 2016.

[20] B. B. Jasani, R. Nanavati, and N. Kabra, “Unusual neonatal presentation of type I leukocyte adhesion deficiency.," J. Clin. Neonatol., vol.3, no. 2, pp. 109-11, Apr. 2014.

[21] A. D. Luster, R. Alon, and U. H. von Andrian, "Immune cell migration in inflammation: present and future therapeutic targets," Nat. Immunol., vol. 6, no. 12, pp. 1182-1190, Dec. 2005.

[22] I. Mitroulis, V. I. Alexaki, I. Kourtzelis, A. Ziogas, G. Hajisheng allis, and T. Chavakis, "Leukocyte integrins: role in leukocyte recruitment and as therapeutic targets in inflammatory disease.," Pharmacol. Ther., vol. 147, pp. 123-135, Mar. 2015.

[23] K. Naran, T. Nundalall, S. Chetty, and S. Barth, "Principles of Immunotherapy: Implications for Treatment Strategies in Cancer and Infectious Diseases.," Front. Microbiol., vol.9, p. 3158, 2018.

[24] T. Kalogeris, C. P. Baines, M. Krenz, and R. J. Korthuis, “Ischemia/Reperfusion.," Compr. Physiol., vol. 7, no. 1, pp. 113-170, 2016.

[25] L. Chen et al., "Inflammatory responses and inflammation-associated diseases in organs.," Oncotarget, vol. 9, no. 6, pp. 7204-7218, Jan. 2018.

[26] K. L. Rock and H. Kono, "The inflammatory response to cell death.," Annu. Rev. Pathol., vol. 3, pp. 99-126, 2008.

[27] A. Dalloul, “B-cell-mediated strategies to fight chronic allograft rejection.," Front. Immunol., vol. 4, p. 444, Dec. 2013.

[28] Z. Szekanecz and A. E. Koch, “Cell Recruitment and Angiogenesis," Kelley Firestein's Textb. Rheumatol., pp. 384-395.e5, Jan. 2017.

[29] E. Perkey and I. Maillard, “New Insights into Graft-Versus-Host Disease and Graft Rejection,” Annu. Rev. Pathol. Mech. Dis., vol.13, no. 1, pp. 219-245, Jan. 2018.

[30] C. Warnke et al., "Natalizumab and progressive multifocal leukoencephalopathy: what are the causal factors and can it be avoided?," Arch. Neurol., vol.67, no. 8, pp. 923-30, Aug. 2010.

[31] T. Wen and M. E. Rothenberg, "The Regulatory Function of Eosinophils.," Microbiol. Spectr., vol. 4, no. 5, 2016.

[32] L. A. Spencer and P. F. Weller, "Eosinophils and Th2 immunity: contemporary insights.," Immunol. Cell Biol., vol. 88, no. 3, pp. 250-6, 2010.

[33] L. A. Doyle, M. Vivero, C. D. Fletcher, F. Mertens, and J. L. Hornick, "Nuclear expression of STAT6 distinguishes solitary fibrous tumor from histologic mimics," Mod. Pathol., vol. 27, no. 3, pp. 390-395, Mar. 2014.

[34] “5-(2-AMINO-L-HYDROXYETHYL)-8-HYDROXY-2-OXOQUINOLINE DERIVATIVES AND OTHER COMPOUNDS AS $\beta 2$-ADRENERGIC AGONISTS," Oct. 2007.

[35] T. A. Malik, "Inflammatory Bowel Disease. Historical Perspective, Epidemiology, and Risk Factors.," Surgical Clinics of North America, vol. 95, no. 6. pp. 1105-1122, Dec-2015.

[36] Y. N. Kim and Y. Jung, "Renal and Urinary Manifestations of Inflammatory Bowel Disease," Korean J. Gastroenterol., vol. 73, no. 5, p. 260, May 2019.

[37] A. Guardiola-Arévalo et al., “Amyloidosis and Crohn's disease.,” Rev. Esp. Enferm. Dig., vol. 103, no. 5, pp. 268-74, May 2011.

[38] J. Leong and B. Fung-Liu, “A Case Report of Nephrotic Syndrome Due to Intake of Certolizumab Pegol in a Patient With Crohn's Disease," Am. J. Gastroenterol., vol. 105, no. 1, p. 234, Jan. 2010.

[39] H. Andersson, I. Bosaeus, S. Fasth, R. Hellberg, and L. Hultén, “Cholelithiasis and Urolithiasis in Crohn's Disease," Scand. J. Gastroenterol., vol. 22, no. 2, pp. 253-256, Jan. 1987.

[40] K. Morita et al., "Crohn's disease following rituximab treatment in a patient with refractory nephrotic syndrome.," CEN case reports, vol. 8, no. 1, pp. 55-60, 2019.

[41] R. J. Shalhoub, “Pathogenesis of lipoid nephrosis: a disorder of T-cell function.," Lancet (London, England), vol. 2, no. 7880, pp. 556-60, Sep. 1974.

[42] B. Szirmay et al., “Elevated urinary orosomucoid excretion as a novel biomarker in Crohn's disease," Eur. J. Clin. Invest., vol. 49, no. 3, p. e13054, Dec. 2018.

[43] M. Hebbar et al., "Association between myelodysplastic syndromes and inflammatory bowel diseases. Report of seven new cases and review of the literature," Leukemia, vol.11, no. 12, pp. 2188-2191, 1997. 
[44] J. Y. Lee et al., "Serum Amyloid A Proteins Induce Pathogenic Th17 Cells and Promote Inflammatory Disease," Cell, vol.180, no. 1, pp. 79-91.e16, Jan. 2020. 